Arq. Bras. Med. Vet. Zootec., v.71, n.4, p.1187-1192, 2019

\title{
Lipidograma e sensibilidade à insulina em éguas Mangalarga Marchador
}

[Lipidogram and insulin sensitivity in Mangalarga Marchador mares]

\author{
E.B.F.R.B. Mello ${ }^{1}$, P.T.L Botteon ${ }^{1 *}$, T.M. Hess ${ }^{2}$, B.F. Spíndola, \\ B.G. Souza ${ }^{1}$, T.L. Barros ${ }^{1}$, B.P.S. Raimundo ${ }^{1}$ \\ ${ }^{1}$ Universidade Federal Rural do Rio de Janeiro - Seropédica - RJ \\ ${ }^{2}$ Colorado State University - Colorado, EUA
}

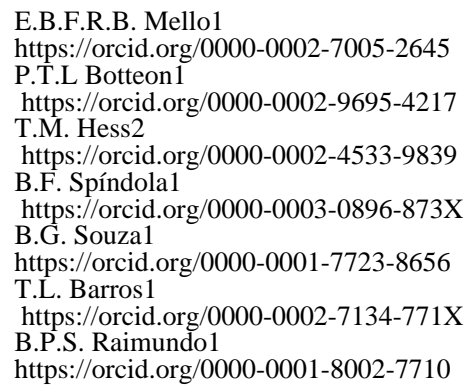

\section{RESUMO}

A dislipidemia é um achado comum, porém não determinante, na síndrome metabólica equina (SME). O objetivo do presente trabalho foi caracterizar a dislipidemia em animais obesos com risco de SME. Para isso, 18 éguas foram alocadas em grupos, de acordo com escore corporal (EC) de 1 a 9: no grupo ideal, animais com EC de 4,5 a 5,5 (n=6), no grupo sobrepeso, com EC de 6 a $7(n=6)$ e no grupo obeso, animais com EC de 7,5 a $9(n=6)$. Coletaram-se amostras de sangue em jejum de concentrado para determinação de triglicerídeos, colesterol total, glicemia e concentração de insulina. Valores preditivos de sensibilidade à insulina (RISQI) e de secreção $\beta$-pancreática (MIRG) foram calculados. O grupo obeso apresentou níveis maiores em relação aos outros grupos de triglicerídeos $(\mathrm{P}=0,001)$ e acima do ideal em concentrações de colesterol $(\mathrm{P}=0,012)$. Não foi observada diferença nas concentrações plasmáticas de glicose $(\mathrm{P}=0,53)$, de insulina $(\mathrm{P}=0,10)$ ou de RISQI $(\mathrm{P}=0,46)$. Houve diferença entre os grupos nos valores de MIRG $(\mathrm{P}=0,048)$, tendo o grupo obeso obtido resultados maiores quando comparado com o grupo ideal. $\mathrm{O}$ aumento do EC foi associado ao aumento das concentrações plasmáticas de colesterol e triglicerídeos, o que caracteriza um estado de dislipidemia e de elevação da secreção das células $\beta$-pancreáticas.

Palavras-chave: equino, trigliceridemia, colesterolemia, disfunção insulínica, obesidade

\begin{abstract}
Increased indicators of fat metabolites are found in Equine Metabolic Syndrome (EMS) subjects, although these parameters are not included in the EMS definition described in the literature and in its diagnosis. The objective of this study was to characterize dyslipidemia in obese insulin resistant mares. 18 mares were allocated in three groups according to body condition score (BCS) in a 1 to 9 scale. In the Ideal group there were animals with BCS 4.5 to $5.5(n=6)$, in the Overweight group, the BCS were 6 to 7 $(n=6)$, and in the Obese group $(n=6), B C S 7.5$ to 9. Concentrate fasting blood samples were taken to determine triglycerides, total cholesterol, glucose, and insulin concentrations in plasma. Insulin sensitivity proxy (RISQI) and $\beta$-pancreatic secretion proxy (MIRG) were calculated from glucose and insulin data. The Obese group had higher triglyceride levels $(P=0.001)$, compared to other groups, and higher total cholesterol compared to the Ideal Group $(P=0.012)$. No differences in plasma glucose $(P=$ $0.53)$, insulin $(P=0.10)$ concentrations and insulin sensitivity (RISQI: $P=0.463)$ were seen among groups. The Obese Group had a higher $\beta$-pancreatic secretion (MIRG: $P=0.048)$ compared to the Ideal Group. The increased BCS was related to the plasma fat metabolites a higher $\beta$-pancreatic secretion.
\end{abstract}

Keywords: equine, triglycerides, cholesterolemia, insulin dysfunction, obesity

Recebido em 18 de dezembro de 2017

Aceito em 11 de dezembro de 2018

* Autor para correspondência (corresponding author)

E-mail: paulobotteon@gmail.com 


\section{INTRODUÇÃO}

A etiologia e a fisiopatogenia da síndrome associada à obesidade e à resistência à insulina, ainda não estão consolidadas. Kronfeld (2003) sugere nomear a síndrome por semelhança aos critérios usados em medicina humana na época. Com o decorrer dos avanços em pesquisa, foi proposto o termo de síndrome de resistência à insulina, em que a resistência à insulina e a obesidade eram tidas como fatores predisponentes para o aparecimento de laminite (Treiber et al., 2006a; Treiber et al., 2006b; Geor, 2008). Essas alterações não se restringiam unicamente ao metabolismo energético dos animais, influenciando o sistema cardiovascular (Bailey et al., 2008) e o ciclo estral em éguas (Vick et al., 2006). A Associação Americana de Medicina Veterinária posteriormente publicou um consenso que denominou de síndrome metabólica equina (SME) o quadro em que os animais apresentavam resistência à insulina, obesidade ou lipodistrofia e histórico de claudicação intermitente ou laminite clínica sem fator predisponente (Frank et al., 2010). Recentemente, os critérios diagnósticos da SME foram revistos, após terem sido considerados imprecisos em um estudo retrospectivo, o qual demonstra que o fenótipo da SME não se restringe ao conjunto de animais com claudicação inespecífica ou laminite clínica, à obesidade e à resistência à insulina $(\mathrm{McCue}$ et $a l ., 2015)$, não sendo fator decisivo à ocorrência dos três sintomas clássicos em conjunto, mas a uma gama de alterações que demonstram disfunção metabólica, semelhante ao observado na síndrome metabólica humana (Obesity, 2012). Observações clínicas indicam que nem todos os animais que apresentam disfunção no metabolismo da insulina relacionada à SME apresentam laminite clínica (Geor et al., 2013).

Em humanos obesos, o quadro de dislipemia é comum, podendo ou não estar relacionado à disfunção insulínica (Obesity, 2012). De forma semelhante, a obesidade está relacionada a quadros de alteração do metabolismo de lipídeos em cavalos (Hughes et al., 2004; McKenzie, 2011). A mensuração de frações de lipídeos na corrente sanguínea permite uma avaliação da mobilização de ácidos graxos do tecido adiposo para utilização como substrato do metabolismo aeróbico de obtenção de energia ou para reesterificação. A incidência de obesidade tem impacto em quadros que cursam com dislipidemia (Hughes et al., 2004; McKenzie, 2011), devido à mobilização insulinodependente.

$\mathrm{O}$ presente estudo visou avaliar animais em diferentes faixas de escore corporal (ideal, sobrepeso e obeso) mediante a análise de lipidograma, glicemia de jejum, concentração de insulina plasmática e nos proxies calculados de sensibilidade à insulina e resposta das células $\beta$ pancreáticas.

\section{MATERIAL E MÉTODOS}

Foram coletadas amostras de soro e plasma de 18 éguas não gestantes e não lactantes, com idade média de 7,5 $\pm 5,5$ anos, clinicamente sadias. $\mathrm{O}$ estudo foi aprovado pela Ceua - 51/2012Fapur/UFRRJ. Os animais selecionados foram distribuídos em três grupos, de acordo com seu escore corporal (Henneke et al., 1983), atribuindo-se variação de 0,5 ponto no EC de modo a evidenciar transição entre categorias. $\mathrm{O}$ grupo ideal - GI $(n=6)$ foi composto por éguas com EC de 4,5 a 5,5; o grupo sobrepeso - GS $(n=6)$, por éguas com EC de 6 a 7 ; e o grupo obeso - GO $(n=6)$, por éguas com EC de 7,5 a 9.

Foram realizadas coletas de alíquotas sanguíneas por punção da veia jugular para determinação de colesterolemia total, trigliceridemia, glicemia e insulinemia de jejum parcial (sem ingestão de concentrado energético) dos animais selecionados, que estavam em jejum há pelo menos oito horas. Para determinação da glicemia, as amostras foram coletadas em tubos de coleta de sangue a vácuo contendo anticoagulante fluoreto de potássio; para determinação da insulinemia, colesterolemia total e trigliceridemia, as amostras foram coletadas em tubos com anticoagulante heparina sódica. Animais que se mostraram indóceis à coleta foram descartados para não haver alterações na glicemia devido à resposta estressogênica do cortisol.

Depois de coletadas, as amostras foram imediatamente acondicionadas em recipiente tipo cooller contendo água e gelo e prontamente centrifugadas a $2000 \mathrm{~g}$, ainda no local de coleta. Foram separadas alíquotas do plasma sobrenadante, as quais foram acondicionadas em microtubos de $1,5 \mathrm{~mL}$. As amostras foram 
transportadas sob refrigeração, em recipiente tipo cooller, até o Laboratório de Pesquisas Clínicas em Grandes Animais (ClinLab) da UFRRJ, onde foram acondicionadas em freezer a $-20^{\circ} \mathrm{C}$ para posterior análise.

As análises de colesterol total, triglicerídeos e glicose foram realizadas utilizando-se kits comercialmente disponíveis (Biosystems ${ }^{\circledR}$ ), de acordo com as recomendações do fabricante. $\mathrm{O}$ material foi processado no Laboratório de Pesquisas Clínicas em Grandes Animais (ClinLab) da UFRRJ, em analisador bioquímico automatizado (A15® - Biosystems $\left.{ }^{\circledR}\right)$.

As concentrações de insulina foram determinadas em duplicata, por meio de radioimunoensaio sanduíche de fase sólida (Insulin(e) IRMA Kit® - Beckman Coulter®) validado para a espécie equina (OBA, 2014 comunicação pessoal - FMVZ/Unesp Botucatu). As análises foram realizadas no laboratório de Biofísica do Departamento de Ciências Fisiológicas da UFRRJ. Os dados obtidos de glicemia e insulina plasmática foram submetidos às equações propostas por Treiber $e t$ al. (2005) para se obterem os valores de predição da sensibilidade à insulina e da resposta secretória de insulina das células $\beta$-pancreáticas à glicose. Para determinação da sensibilidade à insulina, os dados de insulina plasmática foram utilizados na equação que determina o recíproco quadrado inverso da insulina basal (do inglês: RISQI), em que:

$$
R I S Q I=\text { insulina basal }{ }^{-0.5}
$$

Para determinação da responsividade secretória de insulina das células $\beta$-pancreáticas à glicose, os dados de glicemia e insulinemia foram utilizados na equação que determina a razão modificada insulina-glicose (do inglês: MIRG), em que:

$$
M I R G=\frac{\left[800-0.30 x(\text { insulina basal }-50)^{2}\right]}{(\text { glicose basal }-30)}
$$

Para a análise estatística, os dados foram avaliados quanto à distribuição normal pelo teste de Shapiro-Wilk. Dados com distribuição normal, ou que assumiram essa condição após transformação por meio da função $y=\log (y)$, foram testados pelo teste ANOVA one-way, complementatos pelo teste de Tukey. Os dados não normais foram analisados pelo teste de Kruskal-Wallis, com subsequente teste de Dunn (post-hoc). Dados normais foram expressos em média e desvio-padrão, dados categóricos e não normais foram expressos em mediana $\mathrm{e}$ amplitude. Foi respeitado o nível de significância a $5 \%$ em todos os testes utilizados.

\section{RESULTADOS}

Os resultados das análises de colesterol total, glicemia, triglicerídeos e proxie calculada de MIRG, da concentração de insulina e proxies calculadas RISQI e quintilhos de RISQI e MIRG podem ser observados na Tab. 1. Os valores de colesterol total, expresso em $\mathrm{mg} / \mathrm{dL}$, apresentaram tendência de elevação com o escore corporal. Animais com EC ideal, com sobrepeso e obesos apresentaram média e desviopadrão, respectivamente, de $64,83 \pm 14,65$; $87,83 \pm 9,45$ e $106,0 \pm 30,98$, com diferença significativa entre o grupo EC ideal e o grupo obeso (valor $\mathrm{P}=0,012$ ). Os valores de glicemia foram semelhantes entre os grupos (valor $\mathrm{P}=0,53$ ). Foi observada diferença significativa entre os grupos quando avaliadas as concentrações de triglicerídeos (valor $\mathrm{P}=0,001$ ), tendo os animais obesos resultados significativamente maiores em relação aos demais grupos. Os valores de concentração de insulina basal não diferiram significativamente entre os grupos $(\mathrm{P}=0,10)$, assim como do valor preditivo de sensibilidade à insulina (RISQI: $\mathrm{P}=0,46)$. Houve diferença estatística quando avaliada secreção $\beta$-pancreática (MIRG: $\mathrm{P}=0,048)$, tendo os animais obesos resultados significativamente maiores quando comparados a animais classificados em escore ideal. Quando observada a alocação em quintis, os grupos não tiveram diferença na sensibilidade à insulina (quintis de RISQI: $\mathrm{P}=0,30$ ), enquanto os animais obesos apresentaram quintis maiores que animais em escore ideal (quintil de MIRG: $\mathrm{P}=0,010$ ). 
Tabela 1. Média e desvio-padrão dos valores de colesterol total, glicemia, triglicerídeos e proxie calculada de MIRG, seguidos dos valores de mediana e amplitude da concentração de insulina e proxies calculadas RISQI e quintilhos de RISQI e MIRG

\begin{tabular}{lcccc}
\multicolumn{1}{c}{ Parâmetros } & Ideal & Sobrepeso & Obeso & Valor P \\
\hline Colesterol total $(\mathrm{mg} / \mathrm{dL})$ & $64,83 \pm 14,65^{\mathrm{a}}$ & $87,83 \pm 9,453^{\mathrm{ab}}$ & $106,0 \pm 30,98^{\mathrm{b}}$ & 0,012 \\
Glicose (mg/dL) & $88,00 \pm 12,07^{\mathrm{a}}$ & $84,83 \pm 1,472^{\mathrm{a}}$ & $82,67 \pm 6,919^{\mathrm{a}}$ & 0,530 \\
Triglicerídeos $(\mathrm{mg} / \mathrm{dL})_{\text {MIRG U }_{\text {ins }} 2 /\left(10 . \mathrm{L} . \mathrm{mg}_{\text {glic }}\right)}^{8,2 \pm 5,19^{\mathrm{a}}}$ & $4,8 \pm 2,40^{\mathrm{a}}$ & $31,0 \pm 17,82^{\mathrm{b}}$ & 0,001 \\
Insulina $\left.(\mathrm{mUI} / \mathrm{L})_{\text {RISQI (mUI/L }}^{\text {ins }}\right)^{-0,5}$ & $4,69 \pm 2,28^{\mathrm{a}}$ & $6,30 \pm 3,67^{\mathrm{ab}}$ & $9,13 \pm 2,53^{\mathrm{b}}$ & 0,048 \\
QUINT $_{\text {RISQI }}(1-5)$ & $9,11(17,35)^{\mathrm{A}}$ & $7,39(26,84)^{\mathrm{A}}$ & $16,42(21,4)^{\mathrm{A}}$ & 0,100 \\
QUINT $_{\text {MIRG }}(1-5)$ & $0,338(1,363)^{\mathrm{A}}$ & $0,368(0,227)^{\mathrm{A}}$ & $0,284(0,449)^{\mathrm{A}}$ & 0,460 \\
\hline
\end{tabular}

*Médias e desvio-padrão seguidos de mesma letra minúscula na linha não diferem estatisticamente pelo teste de Tukey ao nível de 5\% de significância. Médias e desvio-padrão seguidos de mesma letra grega na linha sofreram normalização por meio da função $y=\log (y)$ e não diferem estatisticamente pelo teste de Tukey ao nível de $5 \%$ de significância. Medianas e amplitudes seguidas da mesma letra maiúscula na linha não diferem estatisticamente pelo teste de Dunn ao nível de $5 \%$.

** RISQI (reciprocal of the square root of insulin): valor preditivo de sensibilidade à insulina; MIRG (modified

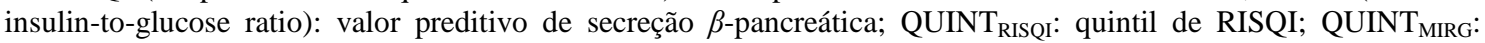
quintil de MIRG.

\section{DISCUSSÃO}

Achados significativos no presente estudo foram maior secreção $\beta$-pancreática e maior concentração de metabólitos da gordura no plasma em animais obesos. Apesar de não ter sido observada diferença estatística entre os quintis na sensibilidade à insulina, (RISQI), animais obesos se encontravam numericamente alocados no primeiro quintil, o que é observado em animais com resistência à insulina, enquanto os animais em EC ideal se encontravam, estatisticamente, no segundo com tendência ao terceiro, e os animais com sobrepeso no terceiro quintil. Os animais do grupo obeso tiveram resultados de quintil de MIRG significativamente maiores em relação aos do grupo ideal, estando no mais alto quintil. Maior resposta da secreção $\beta$-pancreática com manutenção de níveis normais de insulina indica um mecanismo de compensação (Mello, 2012) que, em humanos (Obesity, 2012) e animais, muitas vezes precede a Diabetes mellitus.

Uma das funções da insulina é aumentar a captação tecidual de glicose para formação de reservas energéticas, como glicogênio e depósitos de gordura. Níveis fisiológicos de insulina agem inibindo a ação da lipase hormônio sensível (LHS), responsável pela clivagem de triglicerídeos como via alternativa de manutenção de glicemia a partir do glicerol, liberando, na circulação, ácidos graxos livres
(AGL) (McKenzie, 2011). Insulina atua ainda na na inibição da lipólise (Geor, 2008), e as concentrações de lipídeo circulantes, por sua vez, afetam a sensibilidade à insulina do organismo. Dessa forma, o aumento do escore corporal influencia no quadro de dislipidemia (Hughes et al., 2004; McKenzie, 2011). Os níveis de ácidos graxos circulantes podem ativar mecanismos celulares pró-inflamatórios que têm ação em mecanismos inibidores da sensibilidade à insulina. Assim, os níveis de ácidos graxos circulantes exacerbam o potencial inflamatório, o que resulta em inibição da sensibilidade à insulina. $\mathrm{O}$ aumento de ácidos graxos circulantes funciona como um mecanismo retroalimentador da dessensibilização da função insulínica (Johnson e Olrfsky, 2013). As éguas obesas neste estudo se encontravam em estado dislipidêmico, caracterizado pelo nível mais alto de triglicerídeos (TG) e colesterol, que pode afetar a ação da insulina, levando a uma maior secreção do hormônio para manutenção da euglicemia. McCue et al. (2015) citam o aumento das porções HDL e VLDL colesterol como fatores associados ao fenótipo da SME. O aumento de frações de colesterol vai ter impacto nas concentrações de colesterol total. Cavalos que apresentam fenótipo obeso estão predispostos à dislipidemia (Geor, 2008; Geor et al., 2013; McCue et al., 2015).

O papel da insulina, em níveis considerados homeostáticos, no metabolismo energético está 
ligado ao seu papel na modulação de carreadores GLUT-4, na manutenção da homeostase da glicose, na manutenção das concentrações de glicogênio muscular e hepático, no aumento na $\beta$-oxidação e também à utilização do Acetil-CoA na síntese de ácidos graxos. Os tecidos-alvos responsivos dessa ação da insulina são tecido adiposo, musculatura e fígado (Wilcox, 2005).

Em cavalos, podem ser observadas alterações do comportamento da função insulínica, da leptina e também do metabolismo de lipídeos em indivíduos obesos (Frank et al., 2006). O aumento da concentração de triglicerídeos pode interferir em inúmeras funções fisiológicas, inclusive, de forma parácrina, na ação da insulina, induzindo a um quadro de resistência ao hormônio (McKenzie, 2011). A quantidade de lipídeos influencia na sensibilidade à insulina no miócito e no hepatócito. O quadro está associado ao déficit na oxidação de glicose devido à inibição da piruvato desidrogenase na via glicolítica. Em um primeiro momento, a quantidade intracelular de depósitos de gordura vai afetar a sensibilidade à insulina por defeitos no mecanismo de mobilização de receptores GLUT-4 (Samuel e Shulman, 2012). Quanto mais resistente as células ao efeito da insulina, maiores os níveis secretados para captação de glicose, aumentando a secreção $\beta$-pancreática. No presente estudo, os animais do grupo obeso demonstraram ter depósitos de gordura significativamente maiores que os animais dos demais grupos e valor preditivo de sensibilidade à insulina (RISQI) numericamente inferior aos dos demais grupos, com valor preditivo de secreção $\quad \beta$-pancreática $\quad$ (MIRG) significativamente superior ao grupo ideal. Além disso, os valores de TG dos animais obesos foram significativamente maiores em relação aos dos animais dos demais grupos. Esses achados caracterizam um quadro de hipertrigliceridemia, associado à resistência à insulina nos animais obesos. Essa condição foi relatada anteriormente por diversos autores em estudos com pôneis (Treiber et al., 2006; Bailey et al., 2008; Carter et al., 2009a), mas, até o momento, não foi evidenciada em cavalos da raça Mangalarga Marchador.

\section{CONCLUSÃO}

A elevação do escore corporal dos equinos está associada à elevação das concentrações plasmáticas de colesterol e triglicerídeos, o que caracteriza um estado de dislipidemia e elevação da secreção das células $\beta$-pancreáticas.

\section{AGRADECIMENTOS}

À Fundação de Amparo à Pesquisa do Rio de Janeiro (Faperj E/26/110.894/2012).

\section{REFERÊNCIAS}

BAILEY, S.R. et al. Hypertension and insulin resistance in a mixed-breed population of ponies predisposed to laminitis. Am. J. Vet. Res., v.69, p.122-129, 2008.

CARTER, R.A.; GEOR, R.J.; STANIAR, W.B. et al. Apparent adiposity assessed by standardized scoring systems and morphometric measurements in horses and ponies Vet. J., v.179, p.204-210, 2009.

FRANK, N.; ELLIOTT, S.B.; BRANDT, L.E. et al. Physical characteristics, blood hormone concentrations, and plasma lipid concentrations in obese horses with insulin resistance Am. Vet. Med. Assoc., v.228, p.1383-1390, 2006.

FRANK, N.; GEOR, R.J.; BAILEY, S.R. et al. Equine metabolic syndrome J. Vet. Intern. Med., v.24, p.467-475, 2010.

GEOR, R.J. Metabolic predispositions to laminitis in horses and ponies: obesity, insulin resistance and metabolic syndromes $J$. Equine Vet. Sci., v.28, p.753-759, 2008.

GEOR, R.J.; McCUE, M.E.; SCHULTZ, N. Current understanding of the equine metabolic syndrome phenotype J. Equine Vet. Sci., v.33, p.841-844, 2013.

HENNEKE, D.R; POTTER, G.D.; KREIDER, J.L. et al. Relation between condition score, physical measurements and body fat percentage in mares. Equine Vet. J., v.15, p.371-372, 1983.

HUGHES, K.J.; HODGSON, D.R.; DART, A.J. Equine hyperlipaemia: a review. Aust. Vet. J., v.8, p136-142, 2004.

JOHNSON, A.M.F.; OLEFSKY, J.M. The origins and drivers of insulin resistance Cell v.152, p.673-684, 2013. 
KRONFELD, D.S. Equine syndrome $\mathrm{X}$, the metabolic disease, and equine grain-associated disorders: nomenclature and dietetics. J. Equine Vet. Sci., v.23, p.567-569, 2003.

McCUE, M.E.; GEOR, R.J.; SCHULTZ, N. Equine metabolic syndrome: a complex disease influenced by genetics and environment $J$. Equine Vet. Sci., v.35, p.367-375, 2015.

McKENZIE, H.C. Equine hyperlipidemias Vet. Clin. N. Am. Equine Pract., v.27, p.59-72, 2011.

MELLO, E.B.F.R.B. Adaptação ao exercício em potros puro-sangue inglês de corrida 2012. 54f. Dissertação (Mestrado em Medicina Veterinária) - Programa de Pós-Graduação em Medicina Veterinária. Universidade Federal do Rio de Janeiro, Seropédica, RJ.

OBESITY. World Health Organization. 2012. Available in: <http://www.who.int/topics/ obesity/en/>. Accessed in: 16 Nov. 2017.

RADIN, M.J.; SHARKEY, L.C.; HOLYCROSS, B.J. Adipokines: a review of biological and analytical principles and an update in dogs, cats, and horses Vet. Clin. Path., v.38, p.136-156, 2009.
SAMUEL, V.T.; SHULMAN, G.I. Mechanisms for insulin resistance: common threads and missing links Cell, v.148, p.852-871, 2012

TREIBER, K.H.; KRONFELD, D.S.; GEOR, R.J. Insulin resistance in equids: possible role in laminitis. J. Nut., v.136; p.2094S-2098S; 2006 b.

TREIBER, K.H.; KRONFELD, D.S.; HESS, T.M et al. Use of proxies and reference quintiles obtained from minimal model analysis for determination of insulin sensitivity and pancreatic beta-cell responsiveness in horses. Am. J. Vet. Res., v.66, p.2114-2121, 2005.

TREIBER, K.H.; KRONFELD, D.S.; HESS, T.M. et al. Evaluation of genetic and metabolic predispositions and nutritional risk factors for pasture-associated laminitis in ponies. Am. Vet. Med. Assoc., v.228, p.1538-1545, 2006a.

VICK, M.M.; SESSIONS, D.R.; MURPHY, B.A. et al. Obesity is associated with altered metabolic and reproductive activity in the mare: effects of metformin on insulin sensitivity and reproductive cyclicity. Reprod. Fertil. Dev., v.18, p.609-617, 2006.

WILCOX, G. Insulin and insulin resistance. Clin. Biochem. Rev., v.26, p.19-39, 2005. 